\title{
Public perception and preparedness for the pandemic COVID 19: A Health Belief Model approach
}

\author{
Regi Jose, Meghana Narendran “, Anil Bindu, Nazeema Beevi, Manju L, P.V. Benny \\ Department of Community Medicine, Sree Gokulam Medical College and Research Foundation, Venjaramoodu, Trivandrum, Kerala, India
}

\section{A R T I C L E I N F O}

\section{Keywords:}

COVID-19

Disease outbreaks

Health belief model

Health risk behaviors

Pandemics

Prevention \& control

\begin{abstract}
A B S T R A C T
Background: Coronavirus disease 2019(COVID-19) pandemic is recognized as a major threat to human health in 2020. As the disease is new and spreading quickly, the scientific community is reporting new information daily to address this issue. The public is well informed and is ready to adopt the protective and preventive measures suggested by the authorities.

Aim \& objective: This study is aimed at understanding the perception towards the pandemic using the Health Belief Model (HBM) approach, preparedness measures adopted, opinion and confidence towards various Governmental activities and control measures.

Methods and material: A Cross-sectional survey was conducted among adults across the Kerala state using modified WHO Cosmo protocol and questionnaires implemented through google forms in late April during the preparedness phase of the disease.

Statistical analysis: The results were explained using descriptive and Binary Logistic Regression analysis using SPSS version 22 .

Results: Among the 680 participants, 675 (99.3\%) were aware about COVID-19 and 598 (88\%) had good knowledge. Behavioural change was observed in 638 (93.8\%) and variables in Health Belief Model showed a significant association with behavioural change.

Conclusions: Most people living across Kerala approached the COVID-19 pandemic in a similar manner, irrespective of age, education, or relation to the healthcare field. Perceptions of the community as explained by Health Belief Model was greatly influenced by the primary health care approach by the government and for the subsequent behavioural change.
\end{abstract}

\section{Introduction}

Coronavirus disease 2019(COVID-19) has appeared as one of the most severe pandemics and fatal diseases in human history. ${ }^{1}$ Kerala, the southwestern state of India, with a population of nearly 37 million $^{2}$ had its first COVID- 19 case on January 30th, 2020 and was able to prevent the spread of the disease by strict quarantine and isolation when the rest of the country and world was struggling At the current scenario, the case fatality rate of the world is $7.17 \%$ and in India, $3.27 \% .{ }^{3}$ It is evidenced from previous pandemics that lack of proper knowledge about the disease is associated with negative emotion among people, which can further complicate the attempts of preventing the spread of the disease, ${ }^{4}$ which has a high uncertainty regarding its potential severity and ability to take control over the process by preventive measure. Further, to understand the dynamics of risk perceptions towards the pandemic in a wider perspective, Health Belief Model (HBM) $)^{5}$ was used as a conceptual framework, which has largely been tested empirically, explains, and predicts preventive health behaviour in terms of belief patterns, focusing on the relationship between health behaviours and utilization of health services.

\section{Objectives}

1. To describe the perceptions and behavioural change of the community regarding COVID-19 and control measures in the framework of HBM.

2. To know the preparedness measures adopted by the community during the period of societal controls on free movement (Lockdown)

\footnotetext{
* Corresponding author.

E-mail addresses: regipaul@gmail.com (R. Jose), meghananaren21@gmail.com (M. Narendran), dranilbindu@gmail.com (A. Bindu), nazeemabeevidr@gmail.com (N. Beevi), manjujariharan@yahoo.co.in (M. L), drbennytvm@gmail.com (P.V. Benny).
} 
3. To know the opinion towards various strategies by the government authorities and to find out the association between various HBM variables and the behavioural change outcome.

\section{Materials and methods}

A cross-sectional survey using google forms, was conducted using modified WHO Cosmo protocol questionnaire ${ }^{6}$ Scales for perception and behavioural change and affect had a 7-point ranking scale with the possible responses mentioned in Figs. 1 and 2. Extreme values for Knowledge was no Knowledge to Excellent for COVID 19 and preventive measures, Not Susceptible to Highly Susceptible for perceived susceptibility, Not Severe to Very Severe for severity, Not at all to Very much for self-efficacy, Extremely difficult- Extremely easy for barriers and Not at all-very much so for behavioural change. The answer "neutral" for opinions towards governmental programmes, "declined to answer" and "not sure" for COVID control activities for lockdown preparedness were excluded. To facilitate easier and more instructive interpretation, we transformed the initial 7-point Ranking scale of Perceptions of Knowledge, Susceptibility, Severity, Benefits, Self-Efficacy, Barriers and Practice Intentions into a binary scale. Freidman's test was used to identify the median ranks of each variable and the variables were converted to dichotomous variables using median as cut off.

The approval from the Institutional Ethics Committee (IEC) of Sree Gokulam Medical College \& Research Foundation, Venjaramoodu was obtained prior to data collection. Consent for the study was taken at the beginning of the study through google forms and those who were aged 18 years and over were included for the study. Google form was sent through email to medical students of Sree Gokulam Medical College and their family and telephonic interviews were conducted to ensure participation. Further, they were asked to share the form with their known persons in various districts of Kerala and conduct telephonic interviews. Data was collected from April 28 to 30, 2020. The sample size was calculated assuming the prevalence of knowledge about COVID-19 to be $50 \%$ as it is a new disease and not much data is available. So, considering knowledge among people to be $50 \%$, $95 \%$ confidence level and $5 \%$ relative precision minimum sample size to be studied was calculated using a sample size formula of single proportion to be 384. Considering the limitations of the online survey, we decided to include all the responses we received during the period of data collection. STROBE, for Observational studies in epidemiology guidelines was used for reporting the study. The results were explained using descriptive and Binary Logistic Regression analysis using SPSS version 22.

\section{Results}

The survey was conducted in the early days of COVID-19 pandemic when Kerala had very few cases $^{7}$ and received a total of 700 responses, out of which 680 participants who gave consent were included for analysis. There was nearly equal representation of participants from the healthcare field 325(47.8\%) and others 355(52.2\%). Age distribution was skewed to the left as more youngsters participated in the study and about 346(50.9\%) were less than 25 years. About 409(60.1\%) were females and 450(66.2\%) had completed their graduation, $188(27.6 \%)$ of them were postgraduates and $42(6.2 \%)$ had completed up to 12 years of school education. The awareness about current COVID 19 was 675 (99.3\%) and 638(93.8\%) followed the recommendations from the government. Therefore, we did not do a comparison among healthcare professionals and others and responses from all the participants were analysed as one group.

COVID-19 Specific Behavioural Change: Most of the participants 638 (93.8\%) followed the recommendations by government to combat COVID 19, and 365 (53.7\%) of them updated themselves about COVID 19 several times a day. 647 responded to the question regarding their willingness to vaccinate when it is available and $518(80.1 \%)$ agree that they are more likely to get vaccinated and $129(19.9 \%)$ were against it. There was a significant association between knowledge and (behavioural change), those who had good knowledge had 2.5 odds of good practice [2.5 95\%CI 1.12-5.21] and 2.2 odds of intention to vaccinate 2.2 [95\%CI 1.28-3.63] (Table 1). Majority of the participants were confident that by following hand washing 661 (97.2\%) and by practicing social distancing and lockdown 649 (95.4\%) would help them in prevention of the disease. People's perceptions were collected and explained in the framework of HBM Model and detailed in Fig. 1 and Table 2. Data was collected based on the ranking from one to seven, and

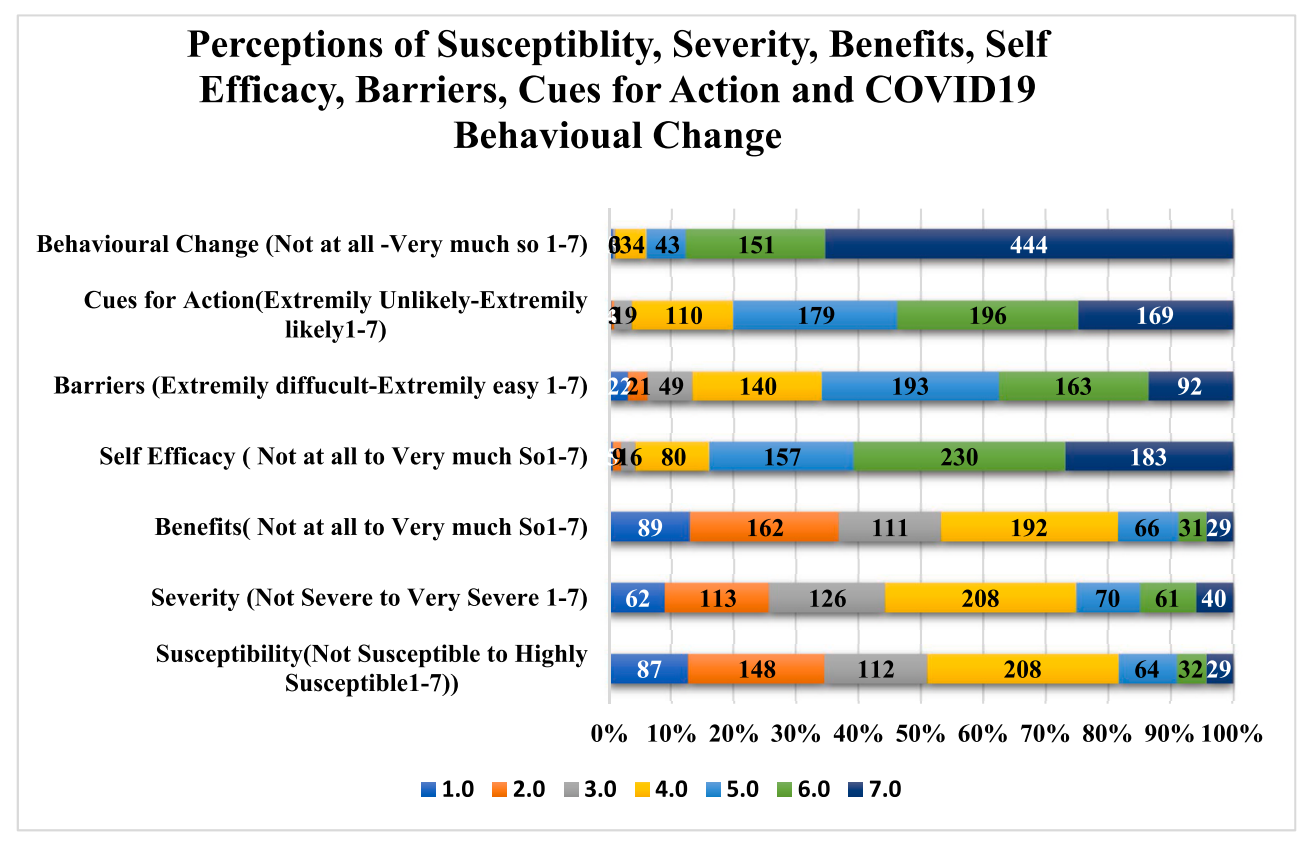

Fig. 1. Perceptions of Susceptibility, Severity, Benefits, Self-Efficacy, Barriers, Cues for Action and COVID19 Behavioural Change Perceived susceptibility was 445 (65.4\%). Perceived severity for getting infected was 379 (55.7\%). Self-efficacy was less among 267 (39.3\%) and 413 (60.7\%) perceived that they were confident in avoiding an infection. Perceived barriers were more among 448 (65.9\%). 


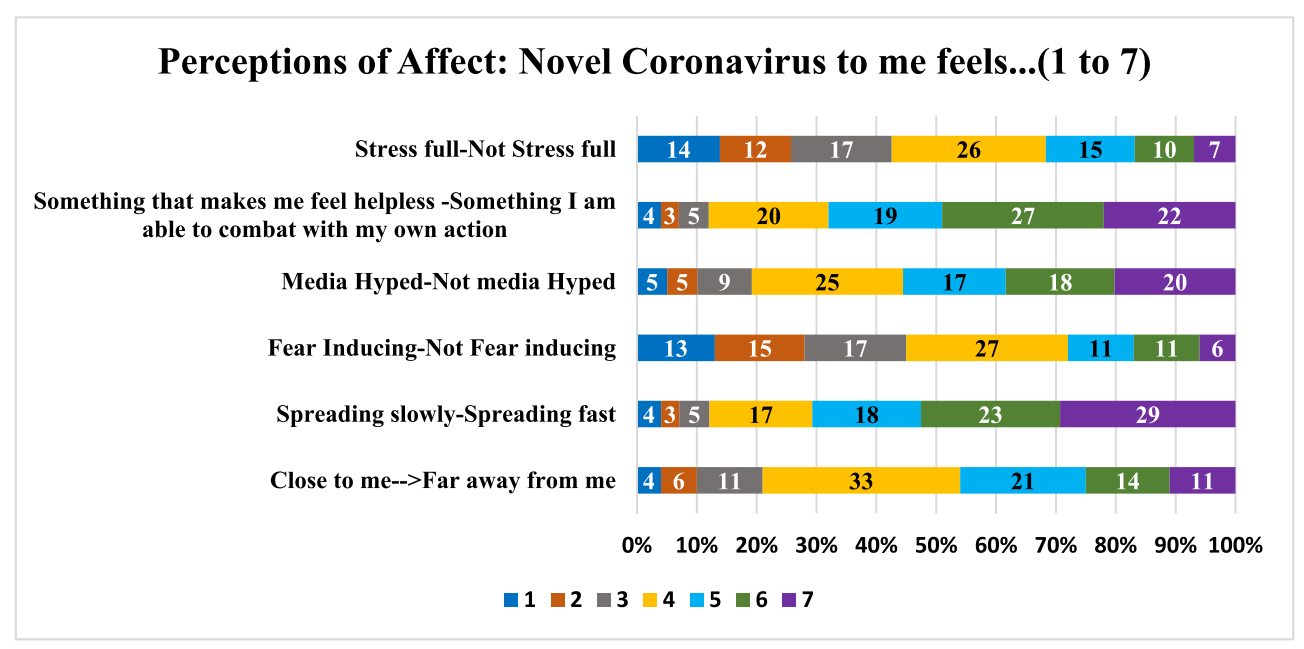

Fig. 2. Perceptions of affect towards COVID 19

Feeling Stressful, helpless, fear inducing, media hype, spread and the disease been felt close to the participants were assessed as affect towards COVID-19.

Table 1

Association of knowledge and Practice (COVID 19 specific behaviour change).

\begin{tabular}{|c|c|c|c|c|c|}
\hline \multirow[t]{2}{*}{ Behavioural Change Variables } & \multicolumn{2}{|c|}{ COVID-19 Knowledge } & \multirow{2}{*}{$\begin{array}{l}\text { Pearson Chi- } \\
\text { Square }\end{array}$} & \multirow[t]{2}{*}{ OR } & \multirow{2}{*}{$\begin{array}{l}\text { P- } \\
\text { Value }\end{array}$} \\
\hline & $\begin{array}{l}\text { Poor } \\
\text { Knowledge }\end{array}$ & $\begin{array}{l}\text { Good } \\
\text { Knowledge }\end{array}$ & & & \\
\hline $\begin{array}{l}\text { I Follow Recommendations by the authorities to avoid Corona virus Infection } \\
(\mathrm{N}=680)\end{array}$ & $82(12 \%)$ & $598(88 \%)$ & 5.829 & $\begin{array}{l}2.457[95 \% \text { CI } 1.159- \\
5.207]\end{array}$ & 0.025 \\
\hline Poor Practice [42 (6.2\%)] & $10(12.2 \%)$ & $32(5.4 \%)$ & & & \\
\hline Good Practice [638 (93.8\%)] & $72(87.8 \%)$ & $566(94.6 \%)$ & & & \\
\hline $\begin{array}{l}\text { If a vaccine becomes available and is recommended for me, I will take it. ( } \mathrm{N}= \\
647 \text { ) }\end{array}$ & $77(12 \%)$ & $570(88 \%)$ & 8.595 & $\begin{array}{l}2.154[95 \% \text { CI } 1.278- \\
3.632]\end{array}$ & 0.004 \\
\hline Less Likely [129 (19.9\%)] & $25(32.5 \%)$ & $104(18.2 \%)$ & & & \\
\hline More Likely [518 (80.1\%)] & $52(67.5 \%)$ & $466(81.8 \%)$ & & & \\
\hline
\end{tabular}

Table 2

Distribution of Health Belief Model Variables in response to COVID-19 Pandemic and its relationship with the Behavioural Change.

\begin{tabular}{|c|c|c|c|c|c|c|}
\hline \multirow{2}{*}{$\begin{array}{c}\text { HBM Variables COVID-19 } \\
(\mathrm{N}=680)\end{array}$} & \multicolumn{2}{|c|}{ COVID-19 Behavioural Change } & \multicolumn{4}{|c|}{ Statistical Inference } \\
\hline & Poor Adherence [42 (6.2\%)] & Good Adherence [638 (93.8\%)] & Chi-Square Tests & P-Value & Crude OR (95\%CI) & MH OR $(95 \% \mathrm{CI})$ \\
\hline \multicolumn{7}{|l|}{ Perceived Susceptibility } \\
\hline Less Susceptible [235 (34.6\%)] & 10 & 225 & 2.29 & 0.179 & $0.57(0.28-1.19)$ & $1.57(0.61-4.02)$ \\
\hline More Susceptible [445 (65.4\%)] & 32 & 413 & & & & \\
\hline \multicolumn{7}{|l|}{ Perceived severity } \\
\hline Less Severe [301 (44.3\%)] & 10 & 291 & 7.59 & 0.006 & $0.37(0.18-0.77)$ & $0.53(0.22-1.27)$ \\
\hline More Severe [379 (55.7\%)] & 32 & 347 & & & & \\
\hline \multicolumn{7}{|l|}{ Perceived Benefits } \\
\hline Less Likely [251 (36.9\%)] & 6 & 245 & 9.84 & 0.001 & $0.27(0.11-0.64)$ & $0.37(0.13-1.02)$ \\
\hline More Likely [429 (63.1\%)] & 36 & 393 & & & & \\
\hline \multicolumn{7}{|l|}{ Self-Efficacy } \\
\hline Less [267 (39.3\%)] & 35 & 232 & 36.46 & 0.001 & $8.75(3.83-20.01)$ & $5.66(2.41-13.29)$ \\
\hline More $[413(60.7 \%)]$ & 7 & 406 & & & & \\
\hline \multicolumn{7}{|l|}{ Perceived barriers } \\
\hline Less [232 (34.1\%)] & 34 & 198 & 43.68 & 0.001 & 9.449 (4.29-20.77) & $6.31(2.78-14.34)$ \\
\hline More [448 (65.9\%)] & 8 & 440 & & & & \\
\hline \multicolumn{7}{|l|}{ Cues for action } \\
\hline Less [136 (20\%)] & 17 & 119 & 11.73 & 0.002 & $2.97(1.55-5.67)$ & $2.58(1.26-5.27)$ \\
\hline More $[544(80 \%)]$ & 25 & 519 & & & & \\
\hline
\end{tabular}

median ranks obtained using Friedman's test was used to divide the responses into two for easy description. About 598 (88\%) participants had an overall good perceived knowledge score and $422(62 \%)$ had good perceived knowledge regarding preventive measures. Perceived susceptibility was more among 445 (65.4\%) and 235 (34.6\%) felt that they were less susceptible for the disease. Perceived severity was less in 301 (44.3\%) and $379(55.7 \%)$ felt that if infected it would be severe for them. A good proportion 429 (63.1\%) felt that they are more likely to avoid infection if they followed the recommendations from the authorities and 251 (36.9\%) felt that they are less likely to benefit from it because no one has a clear idea regarding the pathophysiology and epidemiology of COVID-19. Self-efficacy was less among 267 (39.3\%) and $413(60.7 \%)$ perceived that they were confident in avoiding an infection. Perceived barriers were more among 448 (65.9\%), as most of them updated regarding COVID -19 many times a day through different channels and conflicting information from the COVID infodemic were 


\title{
Community Prepardness Measures
}

\author{
Bought extra medicines \\ Bought extra Regular medication \\ Bought food supplies on a large scale \\ Bought other everyday things on a large scale \\ Bought disinfectants on large scale
}

Stayed away from social events I had planned to attend

Cancelled flights or train rides

Cancelled business trips

Avoided visiting family

Asked friends\& family not to visit me

Imposed restrictions to children

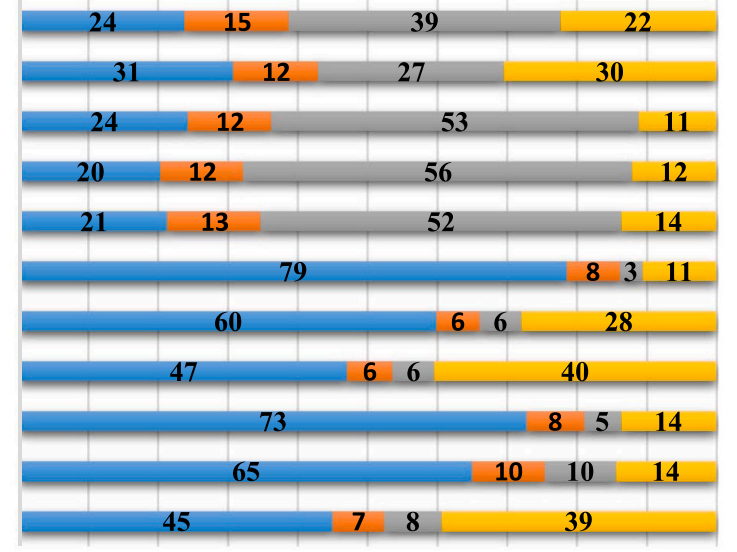

$\begin{array}{lllllllllll}0 \% & 10 \% & 20 \% & 30 \% & 40 \% & 50 \% & 60 \% & 70 \% & 80 \% & 90 \% & 100 \%\end{array}$

I already did that $\quad$ I plan to do that $\quad$ I don't plan to do that $\quad$ Declined to Answer

Fig. 3. Community Preparedness Measures for Societal Control Measures(Lockdown)

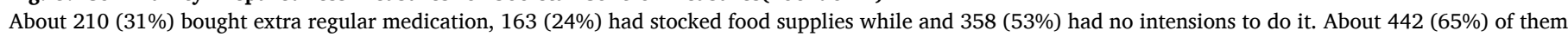
asked friends \& family not to visit them and 495 (73\%) avoided visiting family.

identified as barriers. More than half of the participants 380 (55.9\%) felt that many very important things happen in the world, which the public is never informed about, 379 (55.7\%) felt that politicians usually do not tell the true motives for their decisions which increases the thought barrier for a behaviour change. Opinion regarding various activities imposed by the government were listed and asked whether they could be considered as cues for action for behavioural change and majority 544 (80\%) felt that they had helped them for behavioural change. Binary logistic regression analysis considering behavioural change as outcome variable explains the role of HBM variables in terms of crude and adjusted Odds Ratios(Table 2). Other perceptions of affect mainly stress, feeling helpless, media, fear, and rate of spread of novel coronavirus have been shown in Fig. 2. The Preparedness Measures adopted by the participants towards various guidelines for Lockdown and Social Distancing: About 210 (31\%) bought extra regular medication whereas 265 (39\%) did not plan to buy the same. Among the study participants, $163(24 \%)$ had stocked food supplies while $82(12 \%)$ planned to do so and 358 (53\%) had no intensions to do it. Only 143 (21\%) bought disinfectants on a large scale, $136(20 \%)$ bought other everyday things on a large scale. About 534 (79\%) Stayed away from social events they had planned to attend and $320(47 \%)$ cancelled business trips, 407 (60\%) cancelled flights or train rides. About 442 (65\%) of them asked friends \&

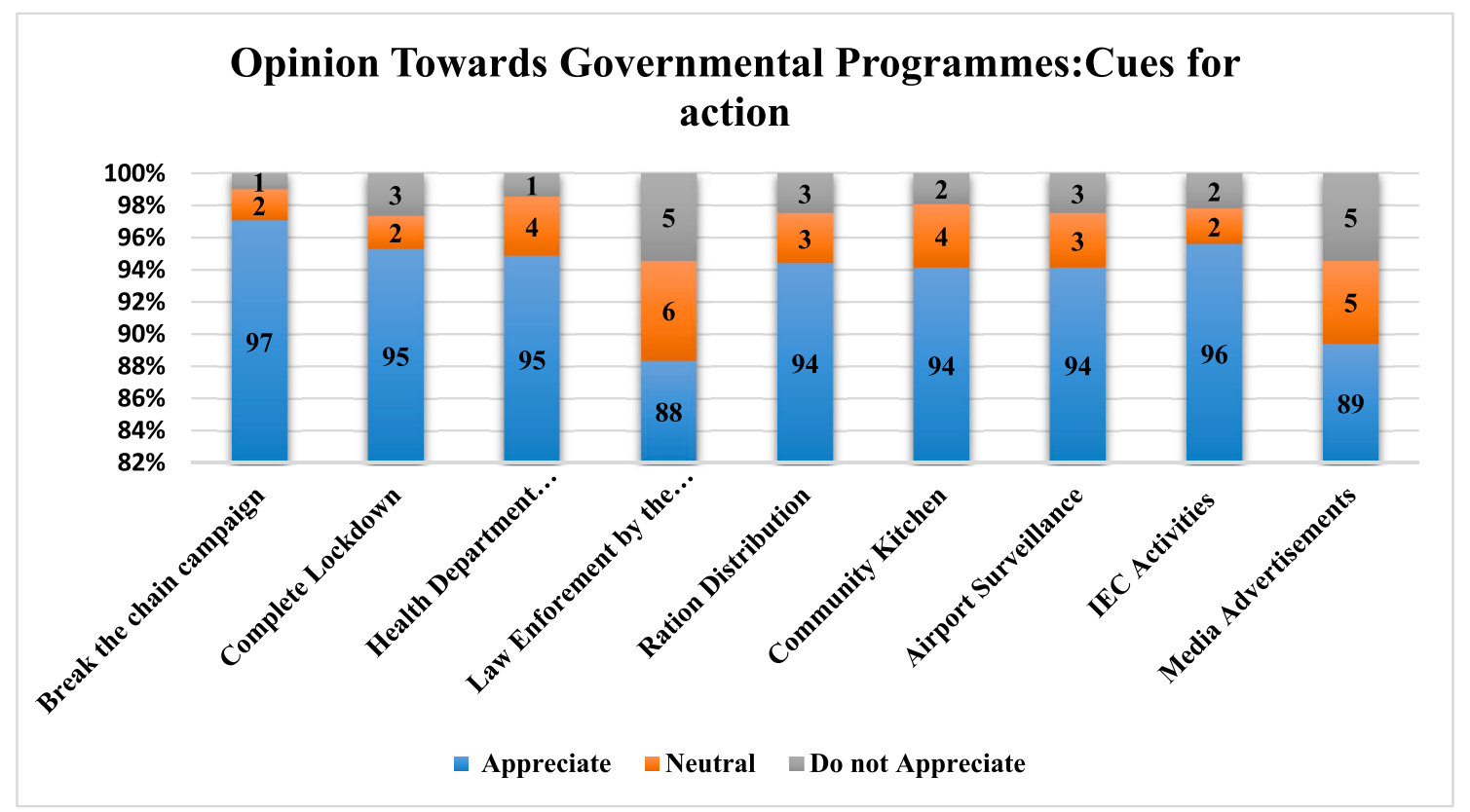

Fig. 4. Public opinion towards various Governmental programmes for COVID 19

The opinion towards various governmental programmes, about 660(97\%), 648(95\%), 645(95\%), 640(94\%) each and 650 (96\%) appreciated for Break the chain campaign, Complete Lockdown, Health Department Preparedness, Ration distribution, Community Kitchen, Airport Surveillance each, and IEC Activities. 
family not to visit them and 495 (73\%) avoided visiting family and 306 (45\%) imposed restrictions on children going outside and play (Fig. 3).

More than $90 \%$ of the participants had appreciation for various programmes like Break the chain campaign, Complete Lockdown, Health Department Preparedness, Ration distribution, Community Kitchen, Airport Surveillance, and IEC activities. Law enforcement by the police and media were less appreciated (Fig. 4).

\section{Discussion}

This study has had nearly equal representation of participants from the healthcare field and other professions. Though there was a good perceived knowledge (88\%) about the current situation of the COVID 19 pandemic, their perceived knowledge for preventive measures (38\%) were poor indicating the need for behavioural change communication. Poor knowledge can be considered as a barrier for behavioural change. Risk perceptions influence individual protective behaviours ${ }^{8}$ but paradoxically, how people perceive risk is not necessarily correlated with the actual risk. This was seen during the influenza pandemic in 2009-10, ${ }^{9}$ where uncertainty and perceived exaggeration were also associated with a reduced likelihood to implement the recommended behaviours. ${ }^{10}$ According to the HBM, increase in perceived susceptibility to a particular health problem would engage in behaviours to reduce their risk of developing the health problem. ${ }^{11}$ Individuals who believe they are at low risk of developing an illness are more likely to engage in unhealthy, or risky, behaviours. The combination of Perceived severity and Perceived susceptibility is referred to as perceived threat ${ }^{12}$ which depend on knowledge about the condition. ${ }^{11}$ The HBM predicts that higher perceived threat leads to a higher likelihood of engagement in health-promoting behaviours. In our study, those who practice hand washing and other measures that prevent the transmission of infection, perceive that contracting COVID 19 would be severe (51\%) and susceptible (60\%), and also most of the elderly having comorbid conditions felt that contracting COVID 19 would be very severe. Therefore, these individuals perceive a given health problem as serious are more likely to engage in behaviours to prevent the health problem from occurring as they feel more susceptible to contract the disease. Perceived benefits in HBM model refers to an individual's assessment of the value or efficacy of engaging in a health-promoting behaviour to decrease risk of disease. ${ }^{13}$ If an individual believes that a particular action will reduce susceptibility to a health problem or decrease its seriousness, then he or she is likely to engage in that behaviour regardless of objective facts regarding the effectiveness of the action. ${ }^{11}$ In this study, most of them felt it was extremely easy to avoid infection if they followed the recommendations set by the government (93.8\%) and followed the same. Perceived barriers refer to an individual's assessment of the obstacles to behaviour change. ${ }^{13}$ The perceived benefits must outweigh the perceived barriers in order for behaviour change to occur. ${ }^{12,13}$ Perceived barriers to taking action include the perceived inconvenience, expense, danger (e.g., side effects of a medical procedure) and discomfort (e.g., pain, emotional upset) involved in engaging in the behaviour. ${ }^{11}$ In this study, perceived barriers were mainly the thought barriers about current situation and they lacked the importance of disease prevention, felt there was much of media hype and secret organizations influenced political decisions which were the barriers in adopting preventive measures. Sudden influx of knowledge through different media (infodemic) made confusion among the public regarding behavioural changes. An overall good knowledge about the disease spread was very much needed to overcome these barriers. The cues of action included trusted programmes by the authorities, events or information from close others, ${ }^{13}$ the media ${ }^{14}$ or health care providers ${ }^{13}$ promoting engagement in health-related behaviours. In this study, more than half $(53.7 \%)$ updated themselves about the current situation several times through media, information from close ones and from healthcare providers which would be necessary for promoting engagement in health-promoting behaviours. Self-efficacy referred to an individual's perception of his or her competence to successfully perform a behaviour. ${ }^{12}$ This recognized the confidence in one's ability to effect change in outcomes which was a key component of health behaviour change. ${ }^{12,15}$ This study adds to our understanding about awareness and promotional activities during pandemic events would have an impact on the pandemic worry, knowledge, behaviour. Additionally, this study goes one step further to consider how Health Belief Model construct during a pandemic event helps to identify and engage in health-promoting behaviour in the population.

\section{Conclusion}

The People of Kerala have acquired adequate knowledge of COVID19, including their susceptibility and severity of the outbreak. Even though a greater proportion had self-efficacy, and had identified barriers, a majority trusted and followed recommendations from the authorities. Most of them had good opinions about the different strategies adopted by the government. The Government could gain the confidence of the community and thus healthy behaviour was observed in the early stages of the pandemic situation.

\section{Recommendation}

This study evolved in response to the needs of communities and populations around the country. The underlying mission was to improve the conditions and behaviours that affect health so that all people can attain it. Perceptions have a great role in behavioural change and all efforts should be focused on improving the perceived susceptibility, severity, benefits, the cues for action, especially in a new pandemic scenario which requires a quick behavioural change. The findings of this study can be used as a baseline for larger in-depth studies to identify factors significantly associated with these problems.

\section{Limitation of the study}

The urgency of the situation incurs some limitations to the study. The use of online panels limited the participation of certain important population groups, including the elderly and disadvantaged population groups such as migrants, homeless people, and other vulnerable groups and literates and one with access to internet and smartphones, laptops or tablets could only be included in the study. So, the results might not be generalizable to the whole state. Phone interviews as a supplement or instead of online panels were used to mitigate the same.

\section{Acknowledgement}

The authors declare that they have no known competing financial interests or personal relationships that could have appeared to influence the work reported in this paper. This research did not receive any specific grant from funding agencies in the public, commercial, or not-forprofit sectors.

\section{References}

1 Wuhan Municipal Health and Health Commission's briefing on the current pneumonia epidemic situation in our city. http://wjw.wuhan.gov.cn/front/web/sho wDetail/2019123108989; 2019. Accessed March 27, 2020.

2 Carlos WG, Dela Cruz CS, Cao B, Pasnick S, Jamil S. Novel wuhan (2019-nCoV) coronavirus. Am J Respir Crit Care Med. 2020;201(4):7-8. https://doi.org/10.1164/ rccm. 2014 P7.

3 World Health Organization. Corona virus. accessed on https://www.who.int/health. topics/coronavirus\#tab=tab_1. Accessed March 27, 2020.

4 Person B, Sy F, Holton K, et al. Fear and Stigma: The Epidemic within the SARS Outbreak. vol. 10. Emerging Infectious Diseases. Centers for Disease Control and Prevention (CDC); 2004:358-363.

5 Rosenstock IM, Strecher VJ, Becker MH. Social learning theory and the health belief model. Health Educ Q. 1988;15(2):175-183.

6 Europe WRO for. COVID-19 Snapshot MOnitoring (COSMO Standard): Monitoring Knowledge, Risk Perceptions, Preventive Behaviours, and Public Trust in the Current 
Coronavirus Outbreak - WHO Standard protocol.COVID-19 Snapshot MOnitoring (COSMO); 2020. Mar 17 [cited 2020 May 29]; Available from: https://www.psych archives.org/handle/20.500.12034/2392.

7 GoK Dashboard. Official Kerala COVID-19 statistics [cited 2020 Jun 1]. Available from: https://dashboard.kerala.gov.in/.

8 Van der Pligt J. Risk perception and self-protective behavior. Eur Psychol. 1996;1: $34-43$.

9 Reintjes R, Das E, Klemm C, Richardus JH, Keßler V, Ahmad A. "Pandemic public health paradox": time series analysis of the 2009/10 Influenza A/H1N1

epidemiology, media attention, risk perception and public reactions in 5 European countries. PloS One. 2016;11, e0151258.

10 Rubin GJ, Amlot R, Page L, Wessely S, Public perceptions, anxiety, and behaviour change in relation to the swine flu outbreak: cross sectional telephone survey. BMJ. 2009;339. b2651.
11 Rosenstock IM. Historical origins of the health belief model. Health Educ Monogr. 1974:328-335. D P.

12 Glanz Karen, Rimer BarbaraK, Viswanath K. Health Behavior and Health Education: Theory, Research, and Practice (PDF). fourth ed. San Francisco, CA: Jossey-Bass; 2008: 45-51. ISBN 978-0787996147.

13 Janz NK, Becker MH. The health belief model: a decade later. Health Educ Q. 1984;11 (1):1-47. https://doi.org/10.1177/109019818401100101. Sep 4 [cited 2020 May 22]; Available from: https://journals.sagepub.com/doi/10.1177/109019818 401100101.

14 A meta-analysis of the effectiveness of health belief model variables in predicting behavior. Health Commun: Vol 25, No 8 [Internet]. [cited 2020 May 22]. Available from: https://www.tandfonline.com/doi/abs/10.1080/10410236.2010.521906.

15 Rosenstock IrwinM, Strecher VictorJ, Becker MarshallH. Social learning theory and the health belief model [Internet]. [cited 2020 May 22]. Available from: https://jo urnals.sagepub.com/doi/10.1177/109019818801500203; 1988. 